\title{
Expression of E-Cadherin and Its Implication in Laryngeal Squamous Cell Carcinoma
}

\author{
Ujjwal Ghosh¹, Suman Ghosh², Anup Kumar Roy ${ }^{3}$
}

\begin{abstract}
1Department of Pathology, Gopiballavpur Super-Speciality Hospital, Jhargram, West Bengal, India. ${ }^{2}$ Department of Pathology, Nil Ratan Sircar Medical College and Hospital, Kolkata, West Bengal, India. ${ }^{3}$ Department of Pathology, Nil Ratan Sircar Medical College and Hospital, Kolkata, West Bengal, India.
\end{abstract}

\section{ABSTRACT}

\section{BACKGROUND}

E-cadherin is essential for cell to cell adhesion and its reduced expression will result in dissemination of cells, cancer metastasis and invasiveness. Lymph-node metastases is an independent prognostic factor in head and neck squamous cell carcinoma. Studies indicate that $4-40 \%$ of patients with laryngeal carcinoma with clinically/radiologically negative neck lymph nodes indeed have occult metastases. So, study of E-cadherin expression would be noteworthy in case of laryngeal squamous cell carcinoma.

\section{METHODS}

An institution based observational cross-sectional study was conducted from February 2017 to July 2018. Patients diagnosed as laryngeal squamous cell carcinoma (DL biopsy/laryngectomy specimen) who did not receive chemo/ radiotherapy were included in the study. Evaluation for grades were done from haematoxylin and eosin stained sections. Formalin fixed and paraffin-embedded blocks were analysed for E-cadherin expression by immunohistochemistry using a scoring system. Data were analysed by Mean \pm Standard Deviation, (Min-Max). The results on categorical measurements are expressed in Number (\%). We have used Student $\mathrm{t}$ test to find the significance of study parameters between the two groups. To find the significance of study parameters on categorical scale between two or more groups we have used Fisher Exact test with the help of statistical software SPSS 18.0.

\section{RESULTS}

A total of 50 laryngeal squamous cell carcinoma cases were enrolled during the entire study period. The age of the patients included in our study varied from 37 years to 77 years with most patients belonging to the age group of 61-70 yrs. 18 out of 50 cases contributing to $36 \%$ belonged to the age group $61-70$ years with the mean age being 57 years. We found a definite statistical correlation between loss of expression of Ecadherin and increasing histological grades of laryngeal squamous cell carcinoma. Positive correlation was also found between neck node metastasis and loss of expression of E-cadherin

\section{CONCLUSIONS}

Through our study we experienced that our ability can be increased to identify those patients who have more aggressive disease by keeping an eye on E-cadherin expression. These patients have more chances of already having occult metastasis at the time of diagnosis and hence may benefit from elective lymph node clearance by neck dissection. Metastasis is a multi-step process and involves many biomolecules. Therefore, only one biomarker will not suffice. A panel of biomarkers can be more effective to predict the metastatic potential and aggressive behaviour of the primary tumour in a better and accurate way.

\section{KEY WORDS}

Laryngeal Squamous Cell Carcinoma, Expression of E-Cadherin, Cervical Lymph Node Metastases

\author{
Corresponding Author: \\ Dr. Suman Ghosh, \\ Associate Professor, \\ Department of Pathology, \\ NRS Medical College \\ 138, AJC Bose Road, \\ Kolkata-700014, India. \\ E-mail:dr_sumanghosh@rediffmail.com
}

DOI: $10.14260 / \mathrm{jemds} / 2019 / 821$

Financial or Other Competing Interests: None.

How to Cite This Article:

Ghosh $U$, Ghosh S, Roy AK. Expression of Ecadherin and its implication in laryngeal squamous cell carcinoma. J. Evolution Med. Dent. Sci. 2019;8(50):3790-3795, DOI: $10.14260 / \mathrm{jemds} / 2019 / 821$

Submission 14-10-2019,

Peer Review 28-11-2019,

Acceptance 04-12-2019,

Published 16-12-2019. 


\section{BACKGROUND}

The most common non-cutaneous head and neck malignancy is laryngeal carcinoma. Squamous cell carcinomas (LSCC) constitute over $90 \%$ of laryngeal malignancies. The major risk factors include tobacco smoking and high consumption of alcohol.[1] The incidence of laryngeal cancer has been reported to be $1.26-8.18$ per 100,000 population in India.[2] The single most adverse independent prognostic factor in head and neck Squamous cell carcinoma is the presence of lymph node metastasis. Data from literature indicate that $4-40 \%$ laryngeal carcinoma patients with clinically or radiologically negative neck lymph nodes, indeed have occult metastases. Adhesion molecules are crucial to maintain cell-cell cohesiveness. Adhesion molecules comprise of five major families: cadherins, integrins, selectins, immunoglobulin gene superfamily (IgSF) and CD44. E-cadherin is a transmembrane glycoprotein of the type 1 cadherin superfamily which is calcium-dependent and is encoded by the CDH1 gene whose location is on chromosome 16q21, and is expressed in most of the epithelial cells. ${ }^{[3]} \mathrm{E}$-cadherin is essential in establishing the cell polarity and in maintaining normal tissue architecture. Ecadherin with its intracellular domain is linked to the actin cytoskeleton through its cytoplasmic-binding partners, the catenins ( $\beta-, \alpha-$, and $\gamma$-Catenin). ${ }^{[4]}$ The E-cadherin and the $\beta$ catenin complex, therefore, functions together to promote cell adhesion. ${ }^{[5]}$ This $\mathrm{E}$ cadherin binding with the protein member of TCF/LEF-1 family by either its cytoplasmic accumulation or its translocation to the nucleus has been associated with various physiological and pathological processes, including tumour progression and metastasis. ${ }^{[6,7]}$

Embryologically the larynx is subdivided into three parts: glottis, subglottis and supraglottis. Glottic carcinomas are more frequently detected early because even small lesions can affect the voice. Growths in supraglottis are more likely to be asymptomatic because tumours do not produce changes in voice until they are significantly advanced. These patients give a history of pain on swallowing, sore throat or otalgia.

Metastasis in lymph nodes worsens the prognosis of the patients with LSCC by decreasing the expected survival up to $50 \%$. Occult metastases on subsequent histological examination have been found in large number of patients with laryngeal carcinoma who had clinically negative lymph nodes on initial assessment. ${ }^{[8,9]}$ The imaging techniques such as ultrasound, computed tomography or magnetic resonance imaging can detect the late macroscopic nodal metastases, but microscopic deposits evade detection. Therefore, it is important to use marker(s) study on the biopsy specimen of the primary tumour that could determine the metastatic behaviour.

\section{Aim}

The study aims at analysing E-Cadherin expression in laryngeal squamous cell carcinoma and to find out relationship between status of E-cadherin expression and neck node metastasis.

\section{Objectives}

1. To diagnose cases of Laryngeal Squamous Cell Carcinomas (LSCC) by histopathological examination.

2. To detect and analyse extent of expression of E-cadherin in different cases of LSCC by Immunohistochemistry and to correlate degree of E-cadherin expression with invasion and metastasis.

\section{METHODS}

An institution based cross-sectional observational study was carried out at Nilratan Sircar Medical College and Hospital (NRS MCH), Kolkata which is a tertiary health-care centre of West Bengal from February 2017 to July 2018 in the Department of Pathology and in collaboration with Department of Ear, nose and throat. After obtaining ethical clearance from Institutional Ethical Committee and informed consent from the study population, the work was initiated. Individuals who presented with hoarseness of voice and subsequently who underwent laryngectomy with or without modified radical neck dissection or direct laryngoscopic biopsy (DL Bx) for diagnostic purpose, were selected as study population. The laryngectomy and DL Bx specimens, as well as lymph nodes obtained from modified radical neck dissection were then sent to the Department of Pathology, NRSMCH for histopathological examination. The total number of cases in the present study is 50 (sample size).Inclusion criterionDiagnosed cases of laryngeal squamous cell carcinoma. Exclusion criteria: Laryngeal Carcinoma other than squamous cell variety and cases who received chemo or radiotherapy.

Consecutive sampling was used. Data were collected using a pretested semi-structured and pre-designed schedule on dependent variables like E-cadherin expression and independent variables like clinico-pathological profile including smoking, macroscopic findings, histological grade and other relevant parameters. Data were collected by interview, record review, observations and laboratory methods including histopathology and immunohistochemistry. Reporting was done by Pathology experts of the same institute.

\section{Histopathology}

$10 \%$ buffered formalin was used to collect all tissue samples and they were processed for routine histopathological examination. For histopathological diagnosis formalin fixed paraffin embedded blocks were cut into $5 \mu \mathrm{m}$ thick sections and stained with haematoxylin and eosin. After histological confirmation of the diagnosis (Laryngeal squamous cell carcinoma), histological grade and lymph node status were determined.

\section{Immunohistochemistry (IHC)}

For immunohistochemistry staining, $3 \mu \mathrm{m}$ thick sections from formalin fixed paraffin embedded tissues were used on poly $L$ Lysine coated slides. IHC was done manually using rabbit monoclonal E-cadherin antibody and the steps mentioned in the kit supplied were followed. Invasive ductal carcinoma of breast (NST) and osteosarcoma were taken as positive and negative control, respectively. Positive findings were evaluated in four fields (1000 cells) under a light microscope at 400X magnification, without knowing the clinical outcome.

\section{Scoring of Immunohistochemistry}

A semi-quantitative evaluation was done which was based on the staining-intensity and distribution (proportion) by using immunoreactive score ${ }^{[10]}$ which combined the intensity and 
proportion score (Immunoreactive score $=$ intensity score $\times$ proportion score). The intensity scores were defined as: $0=$ negative; $1=$ weak; $2=$ moderate; or $3=$ strong, and the proportion scores were defined as: $0=$ negative: $1=<10 \% ; 2=$ $11-50 \%$; $3=51-80 \%$; or $4,>80 \%$ positive cells. The total score was from 0 to 12 . Based on the final score, the immunoreactivity was further divided into three groups: negative immunoreactivity was defined as a total score of 0 , low immunoreactivity was defined as a total score of 1-4, and high immunoreactivity was defined as a total score $>4$. Evaluation of immunostaining of the tumour invasive front was performed by the same method. The stained tumour tissues were scored and also blinded to the clinical patient data.

\section{Statistical Analysis}

Data entry was made in MS excel. We used mean \pm SD, range and percentage for descriptive purposes. Fisher Exact test and Student $t$ test were used to find the significance of study parameters on a categorical scale between two or more groups with the help of SPSS-18. Significance level was considered at $\mathrm{p}$ value $<0.05$.

\section{RESULTS}

A total of 50 laryngeal squamous cell carcinoma cases were enrolled during the entire study period. The age of the patients included in our study varied from 37 years to 77 years with most patients belonging to the age group of 61-70 yrs. 18 out of 50 cases contributing to $36 \%$ belonged to the age group 6170 years and the mean age being 57 years. Out of total 50 cases only 2 cases were female. In our study, among 50 cases of LSCC, $39(78 \%)$ patients revealed history of extended period of smoking, $19(38 \%)$ patients gave history of long-term alcohol intake and $13(26 \%)$ patients were found who were addicted to both smoking and alcohol. Out of 50 LSCC cases in our study, we found 31 cases with tumour location in glottis region (62\%) followed by 17 cases (34\%) and 2 cases (4\%) in supraglottis and subglottis region respectively. Results are obtained by analysis of data and have been presented in the following tables and figures:

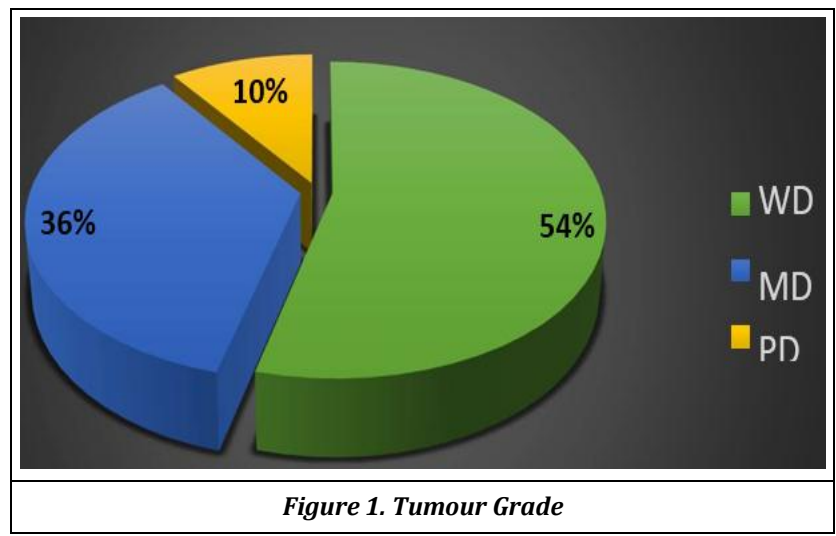

Figure 1. In our study, most of the LSCC were welldifferentiated (WD) (54\%). Rest of the cases were moderatelydifferentiated (MD) (36\%) and poorly- differentiated (PD $10 \%)$ LSCC.
Figure 2. In our study, all the cases below 50 years of age showed positive expression of E-cadherin. Whereas, in the above 50 years age group 16 out of 39 cases showed negative expression of E-cadherin. We observed progressive loss of Ecadherin expression with increasing age. Calculated $p$ value (0.0099) turned out to be statistically significant.

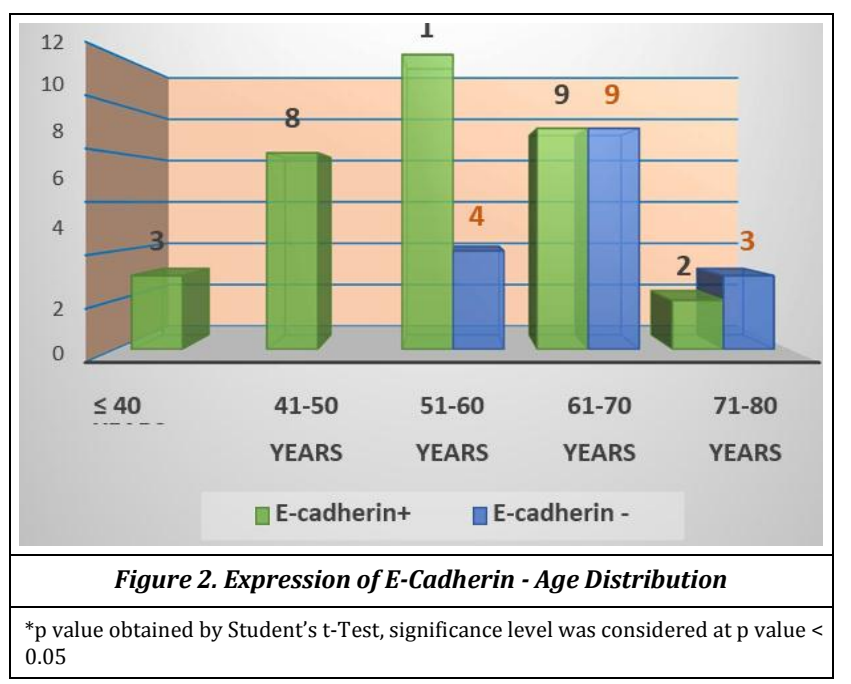

\begin{tabular}{|c|c|c|c|}
\hline Tumour Grade & E-cadherin + & E-cadherin - & Combined \\
\hline Well differentiated & 24 & 03 & 27 \\
\hline Moderately differentiated & 10 & 08 & 18 \\
\hline Poorly differentiated & 0 & 05 & 05 \\
\hline Total & $\mathbf{3 4}$ & $\mathbf{1 6}$ & $\mathbf{5 0}$ \\
\hline Table 1. Tumour Grade and E-Cadherin Expression \\
\hline
\end{tabular}

Table 1. In our study, we tried to correlate tumour grade with expression of E-cadherin. It was observed that all poorly differentiated tumour showed negative expression for Ecadherin (100\% negative expression). In case of moderatelydifferentiated tumour, 8 cases out of 18 (44\%) showed negative expression. Most of the well-differentiated tumour were giving positive expression for E-cadherin. Only 3 out 27 (11\%) cases showed negative expression. Comparison of well differentiated in relation to moderately differentiated and poorly differentiated squamous cell carcinoma combined, calculated Statistically significant $\mathrm{p}$ value obtained by Fisher Exact test was 0.008 (significance level of considered at $p$ value $<0.05$ )

\begin{tabular}{|c|c|c|c|c|}
\hline $\begin{array}{c}\text { Lymph Node } \\
\text { Metastasis }\end{array}$ & $\begin{array}{c}\text { E-Cadherin } \\
\text { Negative }\end{array}$ & $\begin{array}{c}\text { E-cadherin } \\
\text { Positive }\end{array}$ & Combined & $\mathbf{( \% )}$ \\
\hline Present & 12 & 5 & 17 & 34 \\
\hline Absent & 9 & 24 & 33 & 66 \\
\hline Total & $\mathbf{2 1}$ & $\mathbf{2 9}$ & $\mathbf{5 0}$ & $\mathbf{1 0 0}$ \\
\hline \multicolumn{4}{|c|}{0.0059} \\
\hline Table 2. Lymph Node Metastasis and Expression of E-Cadherin \\
\hline $\begin{array}{l}\text { *p value obtained by Fisher Exact Test; significance level was considered at p value } \\
<0.05\end{array}$
\end{tabular}

Table 2. Out of total 50 cases, 17 cases had lymph node metastasis (34\%). Among these 17, 12 cases showed negative expression of E-cadherin (71\%). Calculated $\mathrm{p}$ value was statistically significant.

\section{DISCUSSION}

Integrity of the E-cadherin and catenin complex is essential in maintaining normal adhesion between two cells. In human 
cancer this adhesion is either partially or completely lost with no exception in laryngeal squamous cell carcinoma. Thus, down regulation of E-cadherin plays a key-role in infiltration as well as dissemination or metastasis.

The main observations of this study were that welldifferentiated LSCC expressed E-cadherin often as strongly as normal stratified squamous epithelium, while in moderately differentiated tumour it was expressed in a heterogeneous fashion which includes high, low and even loss of expression. In poorly differentiated tumour, expression was lost. Ecadherin supresses development of tumour by maintaining and increasing adhesion between cells which ultimately inhibits tumour growth by hindering its proliferation. Cell adhesions are weakened by down regulation of E-cadherin which leads to proliferation and eventually metastasis.

\section{Age and Sex}

In our study, we encountered patients of different age groups with minimum age of 37 and maximum age of 77 respectively. Patients mean age at presentation was 58 years. Most of the patients were aged between 61-70 years (18 among the total 50 cases) contributing $36 \%$ of the total sample size. Similar results have been observed by Saedi et al (59.92 years), Lam et al (62 years) and Maier and Tisch et al (57 years).[10-12] Among total 50 patients incorporated in our study, the number of male and female patients were 48 (96\%) and 2 (4\%) respectively and their ratio was $24: 1$. The male preponderance seems to relate to the social habit of smoking which is rare in women in this geographic region. Out of total 690 cases in a similar study of laryngeal carcinoma by Bakshi et al, the number of male and female patients were 647 $(93.77 \%)$ and $47(6.23 \%)$ respectively[13]. In another similar study comprising 66 cases by Goito and Fernandes, 64 (97\%) cases were male.[14]

\section{Risk Factors}

The precise aetiology of laryngeal squamous cell carcinoma is not well understood. Exposure of the mucosa to a wide variety of inhaled and ingested exogenous carcinogenic agents greatly increase the risk of developing these tumours. In our study, among 50 cases of LSCC, 39 (78\%) patients revealed history of smoking, 19 (38\%) patients gave history of long-term alcohol intake and $13(26 \%)$ patients were found who were addicted to both smoking and alcohol. Similar results were found in a study by Bakshi et al, where smoking was associated in $87.8 \%$ cases and consumption of alcohol was a factor which increased the risk of developing LSCC.[13]

\section{Tumour Location}

Location of the tumour inside the larynx is important factor to consider from clinical and prognostic point of view. The supraglottic part of the larynx has profuse lymphatic supply, whereas the glottis has relatively less lymphatics. Subglottic part also is richly supplied by lymphatics. Carcinoma arising in the glottic part produces voice changes, hence even small lesions are detected at early course of the disease. On the other hand, supraglottic and subglottic tumour are difficult to be detected because tumours in those locations do not affect voice. Voice changes are only seen at very advanced stages of disease. Patients complain of sore throat, odynophagia or earache in cases of supraglottic/ subglottic tumours.
Among 50 LSCC cases in our study, we found 31 cases with tumour location in glottis region (62\%) followed by 17 cases (34\%) and 2 cases (4\%) in supra- glottis and subglottis region respectively. However previous studies of Datti et al[15] and Bakshi et al,[13] in which they found supraglottis as commoner site. Glottic tumours with hoarseness of voice, directly lead the clinicians to look for pathology in the vocal cord region and advocating direct laryngoscopy earlier. Whereas symptoms like sore throat, pain on swallowing or otalgia probably do not lead the clinician directly to think that carcinoma of supraglottis or subglottis as a first provisional diagnosis. These symptoms have other more common causes and appear much late in case of carcinoma of supraglottis and subglottis. These may be the reasons which can explain why we found glottis as a commoner site over supraglottis for LSCC. The study result of Larizadeh et al was similar to finding of our study.[16]

\section{Tumour Grade in Respect of E-Cadherin Expression}

We found that, most of the LSCC were well-differentiated (54\%). Rest of the cases were moderately differentiated (36\%) and poorly differentiated (10\%) LSCC. Most of the welldifferentiated tumours were from glottic origin. Among 27 well-differentiated tumours, 24 (89\%) showed positive expression for E- cadherin while only 3 (11\%) showed negative expression. 8 out of 18 moderately differentiated carcinoma gave negative expression for E-cadherin. In case of poorly differentiated carcinoma, it was observed that all tumours showed negative expression for E-cadherin $(100 \%$ negative expression). We found an in- verse correlation between expressions of E-cadherin with degree of differentiation ( $p$ value 0.0002 , statistically significant). These findings were concordant with previous study of Elisabetta et al,[17] Schipper et al[18]and Larizadeh et al[16]; but discordant with Rodrigo et al.[8] and Zvrko et al.[19]

\section{Metastasis to Lymph Nodes and E-Cadherin Expression}

One of the most important prognostic factors which determines the treatment of LSCC, is presence or absence of lymph node metastasis. The detection of lymph node metastasis is difficult at the time of diagnosis. Detection by physical examination and imaging modalities (USG, MRI and CT-scan) is based on the size of lymph nodes. Naturally these methods easily detect macroscopic metastatic lymph nodes but miss those nodes which have microscopic metastasis. Data from literature indicate that $4-40 \%$ patients with LSCC who have microscopic lymph node metastasis escape detection when screened by conventional methods (Physical examination and imaging techniques namely USG, MRI and CTscan)[20-24]. Later on these missed cases are detected when screened histologically. Even USG-guided aspiration/ biopsy identifies clinically occult metastases with a sensitivity of no more than 48 to $76 \%$. That is why a biomarker may become useful to predict metastatic behaviour of the primary tumour. Among total 50 cases included in the study, 17 cases had lymph node metastasis (34\%). Among these 17, 12 cases showed loss of expression of E-cadherin (71\%). When metastasis to cervical lymph nodes was considered against negative expression of E-cadherin (loss), we found an inverse correlation. Calculated p-value (0.0039) was statistically significant. Franchi and colleagues found similar significant correlation between occult lymph node metastases and low E- 
cadherin expression.[25] According to Eriksen et al, Loss of Integrin and E-cadherin which form hemidesmosomes and adherens junction respectively, contribute in predicting risk of metastasis to lymph nodes during diagnosis.[26] However outcome of study of Takes et al. and Liu et al. were discordant to our study result.[27,28]

\section{CONCLUSIONS}

We found a definite statistically significant correlation between loss of expression of E-cadherin and increasing histological grades of laryngeal squamous cell carcinoma. Positive correlation was also found in neck node metastasis and loss of expression of E-cadherin. Our ability can be increased to identify patients who have more aggressive disease by keeping an eye on E-cadherin expression. These patients have more chances of already having occult metastasis at the time of diagnosis and hence may benefit from elective lymph node clearance by neck dissection. Metastasis is a multi-step process and involves many biomolecules. Therefore, only one biomarker will not suffice. A panel of biomarkers can be more effective to predict the metastatic potential and aggressive behaviour of the primary tumour in a better and accurate way. Paucity of cases, limited time period and less scope of follow up were few limitations of our study. Studies incorporating larger sample size, longer duration and proper follow up should rationally increase the probability of accurate prediction about course and behaviour of LSCC.

\section{REFERENCES}

[1] Boffetta P, Hecht S, Gray N, et al. Smokeless tobacco and cancer. The Lancet Oncology 2008;9(7):667-75.

[2] Three-year Report of Population Based Cancer Registries 2009-2011. Bangalore: National Cancer Registry Program (ICMR). Individual registry-wise annexure: 2013: p. 92150.

[3] Pyo SW, Hashimoto M, Kim YS, et al. Expression of Ecadherin, $\mathrm{P}$-cadherin and $\mathrm{N}$-cadherin in oral squamous cell carcinoma: correlation with the clinicopathologic features and patient outcome. J Craniomaxillofac Surg 2007;35(1):1-9.

[4] Chow V, Yuen AP, Lam KY, et al. A comparative study of the clinicopathological significance of E-cadherin and catenins $(\alpha, \beta, \gamma)$ expression in the surgical management of oral tongue carcinomas. J Cancer Res Clin Oncol 2001;127(1):59-63.

[5] Thiery JP. Epithelial-mesenchymal transitions in development and pathologies. Curr Opin Cell Biol 2003;15(6):740-6.

[6] Bánkfalvi A, Krassort M, Végh A, et al. Deranged expression of the E-cadherin/beta-catenin complex and the epidermal growth factor receptor in the clinical evolution and progression of oral squamous cell carcinomas. J Oral Pathol Med 2002;31(8):450-7.

[7] Takeichi M. Cadherin cell adhesion receptors as a morphogenetic regulator. Science 1991;251(5000):14515.
[8] Rodrigo JP, Dominquez F, Alvarez C, et al. Expression of E-cadherin in squamous cell carcinomas of the supraglottic larynx with correlations to clinicopathological features. Eur J Cancer 2002;38(8):1059-64.

[9] Hirohashi S. Inactivation of the E-cadherin-mediated cell adhesion system in human cancers. Am J Pathol 1998;153(2):333-9.

[10] Saedi B, Razmpa E, Sadeghi M, et al. The epidemiology of laryngeal cancer in a country on the esophageal cancer belt. Indian J Otolaryngol Head Neck Surg 2009;61(3):213-7.

[11] Lam KY, Yuen AP. Cancer of the larynx in Hong Kong: a clinico-pathological study. Eur J Surg Oncol 1996;22(2):166-70.

[12] Maier H, Tisch M. Epidemiology of laryngeal cancer: results of the Heidelberg case- control study. Acta Octalarngol Suppl 1997;117(Suppl 527):160-4.

[13] Jaimanti, Panda NK, Sharma S, et al. Survival pattern in treated cases of carcinoma larynx in north India: a 10 year follow up study. Ind J Otolaryngology Head Neck Surg 2004;56(2):99-104.

[14] Goito MC, Fernandes AUR. Risk factors of laryngeal cancer in patients attended in the oral oncology centre of Aracatuba. Braz J Oral Sci 2005;4(13):741-4.

[15] Datti PV, Patel CB, Sayed BA. The incidence of laryngeal cancer in Baroda. Indian J Otolaryngology 1971;23(4):152-62.

[16] Larizadeh HM, Damghani AM, Tabrizchi $H$, et al. Expression of E-cadherin in squamous cell carcinoma of the larynx and its correlation with clinic-pathological features. J Med Sci 2009;9(1):41-5.

[17] Carico E, Radici M, Losito NS, et al. Expression of Ecadherin and $\alpha$-catenin in T1 N0 laryngeal cancer. Anticancer Res 2012;32(12):5245-9.

[18] Schipper JH, Frixen UH, Behrens J, et al. E-cadherin expression in Squamous cell carcinomas of head and neck: inverse correlation with tu- mour dedifferentiation and lymph node metastasis. Cancer Res 1991;51(23 Pt 1):6328-37.

[19] Zvrko E, Mikic A, Jancic S. Relationship of E-cadherin with cervical lymph node metastasis in laryngeal cancer. Coll Antropol 2012;36(Suppl 2):119-24.

[20] Van den Brekel MW, Casteljins JA, Croll GA, et al. Magnetic resonance imaging vs palpation of cervical lymph node metastasis. Arch Otolaryngol Head \& Neck Surg 1991;117(6):666-73.

[21] Gallo O, Boddi V, Bottai GV, et al. The treatment of the clinically negative neck in laryngeal cancer patients. Head \& Neck 1996;18(6):566-72.

[22] Shah JP, Medina JE, Shaha AR, et al. Cervical lymph node metastasis. Curr Probl Surg 1993;30(3):1-335.

[23] Friedman M, Mafee MF, Pacella BL Jr, et al. Rationale for elective neck dissection in 1990. Laryngoscope 1990;100(1):54-9.

[24] Ali S, Tiwan RM, Snow GB. False positive and false negative neck nodes. Head Neck Surg 1985;8(2):78-82.

[25] Franchi A, Gallo O, Boddi V, et al. Prediction of occult neck metastases in laryngeal carcinoma: role of proliferating cell nuclear antigen, MIB-1 and E-cadherin immunohistochemical determination. Clin Cancer Res 1996;2(10):1801-8. 
[26] Eriksen JG, Steiniche T, Søgaard H, et al. Expression of integrins and E- cadherin in Squamous cell carcinomas of the head and neck. APMIS 2004;112(9):560-8.

[27] Takes RP, Baatenburg De Jong RJ, Schuuring E, et al. Markers for assessment of nodal metastasis in laryngeal carcinoma. Arch Otolaryngol Head Neck Surg 1997;123(4):412-9.
[28] Liu M, Lawson G, Delos M, et al. Prognostic value of cell proliferation markers, tumour suppressor proteins and cell adhesion molecules in primary Squamous cell carcinoma of the larynx and hypopharynx. Eur Arch Otorhinolaryngol 2003;260(1):28-34. 